\title{
Drei Zyklen reichen auch langfristig
}

Bei Patienten mit Hochrisiko-Weichteilsarkomen ist eine Behandlung mit drei Zyklen Anthrazyklin plus Ifosfamid einer Behandlung mit fünf Zyklen auch im 10-Jahres-Follow-up nicht unterlegen.

Die Standardtherapie bei lokalisierten Hochrisiko-Weichteilsarkomen umfasst Operation und Bestrahlung. Häufig kommt noch eine adjuvante Chemotherapie dazu. Studiendaten hatten gezeigt, dass die Patienten von der neoadjuvanten Chemotherapie mit drei Zyklen Anthrazyklin/Ifosfamid profitieren. Das 5-JahresGesamtüberleben (OS) war nicht schlechter als unter einer 5-Zyklen-Therapie.

Die Ergebnisse zum medianen 10-Jahres-Follow-up (103-135 Monate) bestätigen die Nichtunterlegenheit der Behandlung mit drei Zyklen. In dieser Zeit waren 58 Patienten der 3-Zyklen-Gruppe und 65 Patienten der 5-Zyklen-Gruppe gestorben. Die Rate für das 10-Jahres-OS lag bei 64 bzw. 59\%. 3-Zyklen waren 5-Zyklen auch nach median zehn Jahren nicht unterle- gen. Die Hazard Ratio (HR) beim Endpunkt OS betrug 0,92 (3 vs. 5 Zyklen), beim progressionsfreien Überleben 1,04. Die besten Ansprechraten erzielten Patienten mit undifferenziertem pleomorphem Sarkom, die schlechtesten Patienten mit Leiomyosarkom. Die Prognosen waren entsprechend besser oder schlechter. Nur wenige Patienten, die bereits fünf Jahre überlebt hatten, entwickelten in den nächsten fünf Jahren ein Rezidiv.

Ob bei bestimmten Subtypen eine entsprechend den histologischen Befunden maßgeschneiderte neoadjuvante Chemotherapie der 3-Zyklen-Therapie überlegen ist, wird derzeit überprüft.

Barbara Kreutzkamp

Gronchi A et al. Short, full-dose adjuvant chemotherapy (CT) in high-risk adult soft tissue sarcomas (STS): long-term follow-up of a randomized clinical trial from the Italian Sarcoma Group and the Spanish Sarcoma Group. Ann Oncol. 2016; 27(12):2283-8

\section{Neutropenie bei myelosuppressiver Chemotherapie Risiko vor jedem Zyklus neu ermitteln}

Bisher entwickelte Modelle zur Risikoermittlung einer Chemotherapie-induzierten Neutropenie (CIN) bzw. einer febrilen Neutropenie (FN) beruhen auf patienten-, behandlungs- und krankheitsbezogenen Variablen, die nur zu Beginn der Chemotherapie erhoben werden. Ein

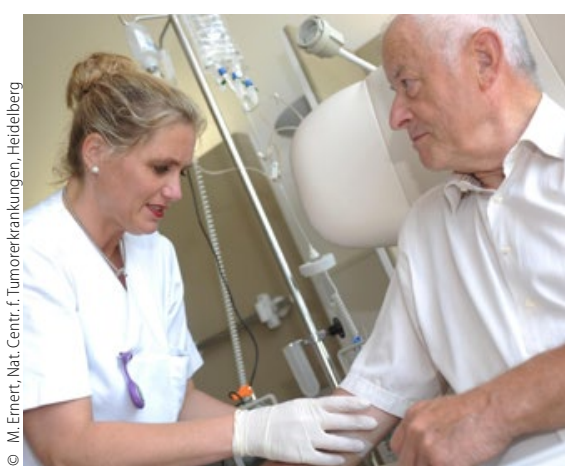

Das Neutropenierisiko unter einer Chemotherapie lässt sich nun besser abschätzen. neues dynamisches Modell ermöglicht nun die individuelle Abschätzung des Neutropenierisikos vor jedem Chemotherapiezyklus. Datenbasis für das Modell war die MONITOR-GCSFStudie, die unter Real-life-Bedingungen stattfand. Die Prophylaxeintensität in den vorangegangenen Zyklen war dabei eine zusätzliche, belastbare Prädiktionsvariable.

In der unter den Bedingungen der täglichen Praxis durchgeführten MONITOR-GCSF-Studie wurden Behandlungsverläufe und Therapieergebnisse von Patienten mit soliden und hämatologischen Malignitäten im Stadium 3 oder 4 unter einer myelosuppressiven Behandlung untersucht. Für die Prävention einer CIN bzw. einer FN kam in dieser Studie das GCSF-Biosimilar EP2006 zum Einsatz. EP2006 ist wirkungsgleich mit dem Referenzprodukt Filgrastim. Wie bei den statischen Modellen werden auch für das

\section{kurz notiert}

Forschungsstandort Deutschland

Antikörper gegen Strahlenfibrose +++ Wissenschaftler vom Deutschen Krebsforschungszentrum setzten bei Mäusen einen Antikörper gegen den Bindegewebe-Wachstumsfaktor (CTGF) erfolgreich zur Behandlung einer Strahlenfibrose ein. CTGF wird als zentraler Signalgeber bei der Entstehung der Lungenfibrose angesehen. Die Therapie mit dem CTGFAntikörper schützte bis zu $80 \%$ der Tiere vor einer Fibrose. Bei einem Start der Behandlung 16 Wochen nach der Bestrahlung machte die CTGF-Blockade eine fibrotische Umwandlung wieder rückgängig [Bickelhaupt S et al. J Natl Cancer Inst. 2017;109(8):djw339].

Identifizierung von Biomarkern +++ Biophysiker der Ruhr-Universität Bochum haben ein neues Verfahren etabliert, mit dem sie Biomarker zur Diagnose verschiedener Krebsarten identifizieren können. Hierfür verwendeten sie eine spezielle Form der Infrarotspektroskopie. Anders als bei den derzeit in der Pathologie angewandten markerbasierten Verfahren bleibt das Gewebe dabei unbeschadet und kann anschließend besser detaillierten Proteinanalysen unterzogen werden [Großerueschkamp F et al. Sci Rep. 2017;7:44829]. red.

dynamische Modell Angaben u.a. zu Anamnese, Risikofaktoren und Therapieintensität benötigt, darüber hinaus zusätzliche Informationen über mögliche CIN-/FN-Zwischenfälle und den Einsatz von GCSF-Präparaten in den vorangegangenen Zyklen, die Zyklusanzahl sowie behandlungszentrumsspezifische Charakteristika. Einige der in aufwendigen statistischen Verfahren identifizierten Prädiktoren fanden sich in mehreren der benutzten Einzelmodelle wieder.

Neben bekannten Einflussfaktoren wie Patientenalter oder Chemotherapieregime traf dies auch für die Prophylaxeintensität zu: Sowohl im patienten- als auch im zyklusbezogenen Modell erwies sich eine Unterprophylaxe als klinisch unsicher, während eine GCSF-Überprophylaxe möglicherweise nützlich ist - auch bei nur mäßig- oder geringgradig myelosupressiven Regimes.

Barbara Kreutzkamp

Aapro M et al. Predictive modeling of the outcomes of chemotherapy-induced (febrile) neutropenia prophylaxis with biosimilar filgrastim (MONITOR-GCSF study). Ann Oncol. 2016;27(11): 2039-45. 